DIVISION OF THE HUMANITIES AND SOCIAL SCIENCES

CALIFORNIA INSTITUTE OF TECHNOLOGY

PASADENA, CALIFORNIA 91125

ON THE INFORMATIONAL INEFFICIENCY OF DISCRIMINATORY PRICE AUCTIONS

Matthew O. Jackson

California Institute of Technology

Ilan Kremer

Stanford University

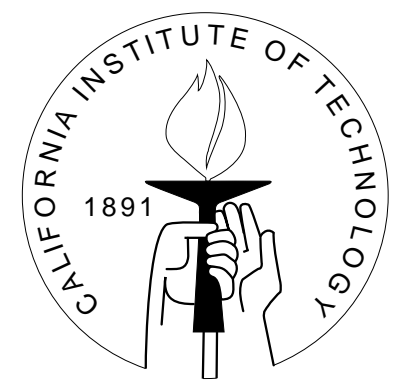

SOCIAL SCIENCE WORKING PAPER 1191 


\title{
On the Informational Inefficiency of Discriminatory Price Auctions
}

\author{
Matthew O. Jackson \\ Ilan Kremer
}

\begin{abstract}
We analyze bidding behavior in large discriminatory price auctions where the number of objects is a non-trivial proportion of the number of bidders. Bidders observe private signals that are affiliated with the common value. We show that the average price in the auction is biased downward from the expected value of the objects, even in the competitive limit. In particular, we show that conditional on relatively low signals, bidders bid the expected value of the objects conditional on their information and winning; while bids at higher signals flatten out and are below the expected value conditional on winning.
\end{abstract}

JEL classification numbers: D44, C72, D41, G14

Key words: Auction, Competition, Discriminatory Auction, Asymptotic Efficiency, Efficient Markets, Efficient Market Hypothesis 


\title{
On the Informational Inefficiency of Discriminatory Price Auctions *
}

\author{
Matthew O. Jackson Ilan Kremer
}

\section{Introduction}

Efficient Market Hypotheses posit that markets aggregate the information held by different agents in an economy and that prices come to reflect the expectation of the value of assets based on this information. This informational role of prices is important for a variety of reasons including the central one that it aids in efficient investment decisions. As such, it is important that we understand the conditions under which efficient market hypotheses hold.

In this paper we examine price behavior in discriminatory auctions with many agents and many goods, with a focus on the relationship between equilibrium prices and the true value of the goods. There are at least two reasons for being interested in auctions when examining the issues mentioned above. The first is that auctions are widely used in the real world. The second is that much of the strategic reasoning that underlies the analysis of auctions is present even in markets that are not formally organized as auctions. In terms of our attention to discriminatory auctions: while the existing literature focuses on the uniform price auction; there are good reasons why we should also examine the discriminatory auction. The discriminatory auction format is very widely used, including many formally structured treasury auctions. Moreover, discriminatory auctions are a good model of price discrimination in more loosely organized markets, as in many markets there is no single clearing price and in a sense, "winning" agents pay their bids.

We examine a discriminatory auction in which many units are being sold to many agents who each demand a single unit whose value is common across all bidders. We are interested in the competitive limit and hence take the limit as we increase the number of bidders. Our characterization of prices comes from understanding bidding behavior and the expected gains that agents have as a function of their signals. We find that bidders who are uncertain of whether they will win an object end up competing away their surplus so that their conditional expected utility is zero. That is, agents whose signals are below a certain threshold bid the expected value of the object conditional on

${ }^{*}$ We are grateful for financial support from the National Science Foundation under grant SES-9986190. 
their signal and winning. However, there are also agents whose signals are high enough that they are certain to get an object, and these agents extract surplus even in the limit. For signals above this threshold, the bidding strategy flattens out, as agents are (nearly) certain that they will win an object, and so agents observing higher signals have positive expected utilities. This leads to a distinct price pattern, prices deviate from Rational Expectations Equilibrium or Walrasian prices (or uniform price auction prices) in a particular way. When we integrate over all realizations, the average price is biased downward from true value. When conditioning on low values we find that average price tends to be too high and conversely when the value is high the average price tends to be too low.

Before presenting our results, let us discuss the contribution of our work relative to a few papers. The most closely related papers examine information aggregation in common value auctions. Wilson (1977) and Milgrom $(1979,1981)$ provided important results characterizing when equilibrium prices come to reflect the expected value of an asset. They examined common-value auctions with a fixed number of units of the asset for sale and a growing number of bidders, and identified conditions under which prices converge to the expected value of the asset as if the economy had the information of all bidders. Their results might be interpreted as "bad news" for efficient market hypotheses in the sense that the necessary and sufficient condition on the structure of information is a very strong one and unlikely to be met in most market settings of interest.

In an important recent paper, Pesendorfer and Swinkels (1997) (PS hereafter) showed that the news is much better if one considers settings where the number of units of the asset for sale grow in addition to the number of bidders. They showed that the limiting price of a uniform price auction converges to the expected value of the asset as if the economy had the information of all bidders under quite weak assumptions on the information structure. As many markets of interest, such as Treasury auctions, have both large quantities of the asset for sale as well as large numbers of bidders, this is "good news" for market efficiency. Apart from the auction format, the model we presents is similar to PS.

As the results of Wilson (1977) and Milgrom (1979, 1981) hold for a wide variety of auction formats, ${ }^{1}$ one might wonder whether the PS results do as well. We demonstrate that, unfortunately, the price-efficiency result of Pesendorfer and Swinkels (1997) critically depends on using the uniform price auction format. Specifically, we show that information aggregation fails in the context of one of (if not the) most prominent auction format: namely the discriminatory price auction. The additional results that we provide characterize exactly how the bidding strategies and average price in a discriminatory are biased relative to the true value of an object; which, of course, is necessarily new as previous papers have focused on cases where prices come reflect true value.

\footnotetext{
${ }^{1}$ See Bali and Jackson (2002) and Jackson and Kremer (2003ab) for results on the equivalence of auction formats with fixed numbers of objects for sale but large numbers of bidders.
} 


\section{Model}

We examine a framework that is similar to PS, but with the notable difference that we examine discriminatory auctions rather than uniform auctions.

We examine a sequence of discriminatory auctions with an increasing number of bidders. The sequence is indexed by $n$, the number of agents in the economy. A nonrandom supply of $k_{n}$ identical indivisible goods is to be sold in economy $n$. The highest $k_{n}$ bidders are each awarded a single unit and each pay his or her bid. The other bidders pay nothing. Ties are broken by uniform randomization among the tied bidders.

We assume that:

(A1) The supply of goods is proportional to the number of bidders: $\frac{k_{n}}{n} \in(0,1)$ for all $n$ and $\frac{k_{n}}{n} \rightarrow \gamma \in(0,1)$.

Each agent values a single unit at the same (common) value $v$ and puts no value on further objects. This value is unknown to bidders and is distributed on $[0,1]$, as described by a continuous density function $f$.

(A2) The density function $f$ is continuous and positive on $[0,1]$.

Agents are risk neutral and agent $i$ receives a payoff of $v-b_{i}$ if he or she receives an object with a bid of $b_{i}$ and the value turns out to be $v$, and 0 otherwise.

Before bidding, an agent $i \in\{1, \ldots, n\}$ in economy $n$ observes a private signal, the realization of a random variable $S_{i}$, that takes on values in $[0,1]$. The $S_{i}$ 's are independently and identically distributed conditional on $V$. This conditional distribution of $S_{i}$ given $V$ is described by a conditional density function $g_{S_{i} \mid V}(s \mid v)$.

A standard affiliation condition ensures the existence of increasing and symmetric equilibria in both the discriminatory and uniform price auctions. Specifically, we follow PS in assuming such an affiliation condition in addition to some continuity and differentiability conditions:

(A3) $g_{S_{i} \mid V}(s \mid v)$ is continuous in $(s, v)$ and continuously differentiable in $v$ for all $s$ and $v$.

The continuity of the conditional density function limits the potential information that can be contained in any given signal, while the differentiability is for technical convenience.

(A4) for every signal, $s$ there exists a value $v$, so that $g_{S_{i} \mid V}(s \mid v)>0$. 
(A4) together with (A3) ensures that each signal has some non-trivial probability of being observed, which is also important in putting an upper bound on the information content of signals.

(A5) $g_{S_{i} \mid V}(s \mid v)$ satisfies the Strict Monotone Likelihood Ratio Property (henceforth, SMLRP):

$$
\frac{\partial}{\partial v} \frac{g_{S_{i} \mid V}(s \mid v)}{g_{S_{i} \mid V}\left(s^{\prime} \mid v\right)}>0
$$

for all $s_{i}>s_{i}^{\prime}$ in $[0,1]$ and all $v \in[0,1]$, and this partial derivative is continuous.

Note that we change the standard weak inequality in the MLRP condition to be a strict one, and we add continuity for technical convenience. This strengthening to be strict is essential to our results. For instance, if signals are independent of the common value (as allowed under a weak MLRP) then we get no correlation between prices and values and prices are simply the unconditional expected value of an object.

Let $G_{S_{i}}(s)$ denote the unconditional distribution of $S_{i}$ and $g_{S_{i}}$ be the corresponding density, $Y^{n}$ denote the $k_{n}$-th order statistic of the signals, and $Y_{-i}^{n}$ denote the $k_{n}$-th order statistic of the signals excluding $i$ 's signal. Let $G_{V \mid S_{i}}(v \mid s)$ denote the distribution of $V$ conditional on observing a signal $S_{i}$. Let $s^{*}(v)$ be the $s$ such that

$$
G_{S_{i} \mid V}(s \mid v)=1-\gamma
$$

As the equilibrium bidding strategy turns out to be increasing, this is the asymptotic cutoff signal for getting an object, conditional on $V=v$. Under our assumptions $s^{*}(v)$ is well-defined and increasing in $v$, and $s^{*}(1)<1$.

For $s \geq s^{*}(0)$, let $v(s)$ be such that $G_{S_{i} \mid V}(s \mid v(s))=\gamma \cdot{ }^{2}$ Thus, $v(s)$ is the value for which $s$ would just be on the margin of getting an object in an increasing equilibrium in the limit. Note that $s^{*}(v(s))=s$ for $s \in\left[s^{*}(0), s^{*}(1)\right]$, so this is the inverse function of $v(\cdot)$.

We assume that:

$$
G_{V \mid S_{i}}(v(s) \mid s)>0 \text { for all } s \text { such that } v(s)>0 .
$$

(A6) limits the information that a signal contains about where the value might lie. While this is not a condition directly on the primitives $f_{V}$ and $g_{S_{i} \mid V}$, it is implied if, for instance, one requires in conjunction with our other conditions that $g_{S_{i} \mid V}$ have full support for each value of $V$.

\footnotetext{
${ }^{2}$ For $s \geq s^{*}(1)$, follow the convention of setting $v(s)=1$.
} 


\section{The Main Result}

In this setting there exists a symmetric pure-strategy Bayesian equilibrium for every $n$. A direct extension of the results of Milgrom and Weber (1982) for first price auctions implies that we can find such a symmetric pure bidding strategy that is increasing in the bidder's signal, which we denote by $b_{n}(s)$ (see the appendix for an explicit characterization). We are interested in the limiting properties of this bidding strategy as $n$ becomes large. Let $h(s)$ be the expected value of an object conditional on the event that (in the limit) an agent wins the auction with a signal $s$ under the symmetric increasing strategies. So,

$$
h(s)=E\left(V \mid S_{i}=s, v(s) \geq V\right) .
$$

Our main result is that:

Theorem 1 If A1 through A6 hold, then

$$
b_{n}(s) \rightarrow \min \left\{h(s), h\left(s^{*}(1)\right)\right\} .
$$

Thus, the expected price in the auction converges to a level that is below the expected value of the asset.

The theorem actually characterizes the limiting bidding strategies, well beyond simply saying that the price converges to be below the expected value. In particular, bids for relatively low signals converge to be the expected value conditional on winning, and bids for relatively high signals converge to a cutoff price, which is the bid that corresponds to a signal such that any signal above this level is sure to get an item in the limit. The second part of this characterization is easy to see, while the first part of the theorem that bids for low signals converge to expected value - is much more difficult to prove. We provide a proof in the appendix.

Let us offer a couple of observations about the price pattern that the above theorem implies.

- The average price would not converge to the value of the object even if all agents bid according to $h(s)$. To see this consider the case where $V$ is close to zero and focus on agents who observe the highest signals. They will bid an amount significantly above zero, and the average price will exceed the asset's value. Conversely, conditioning values that are close to one, prices will be too low. This last effect is enhanced by the fact that the bidding function flattens.

- The discriminatory auction leads to a lower expected price than the uniform price auction, as Pesendorfer and Swinkels (1997) have shown that the price of the uniform price auction converges to the expected value of the objects in the limit. ${ }^{3}$

\footnotetext{
${ }^{3}$ The same holds in a wide variety of private value settings too, as shown in Jackson and Kremer (2003a).
} 
- In the limit, the increasing nature of the equilibrium means that it is "revealing" in the sense that someone who knows the structure of the bidding functions and sees the average price can correctly forecast the value of the asset. However, this is a weak form of "revelation". Most importantly, such a weak form of revelation does not capture the spirit of efficient market hypotheses and does not identify mechanisms that aggregate information. The idea is that the market should come to mold prices in ways so that they reflect the value of assets; not that an observer through the daunting task of observing the appropriate bids and inverting them to deduce signals can unravel the information present in the economy.

To better understand our results, consider the following example.

\section{Example 2}

- The number of units for sale is $k_{n}=n / 2$.

- The value of the good $v$ is distributed uniformly on $[0,1]$.

- Conditional on $v$, signals are distributed uniformly on $[0, v] .{ }^{4}$

In the uniform price auction as PS have shown that prices converge to the true value of the asset $V$. In this case, it is easy to see that the bids in the symmetric equilibrium of the uniform auction converge to being twice the signal $\left(2 S_{i}\right)$. As the median signal sets the price, and this median converges in probability to $S_{i}=v / 2$, it follows that the price converges to the value.

The symmetric equilibrium bids in the discriminatory price auction (1) are harder to handle for a finite $n$, as $v(s, s)$ involves a non-trivial computation. However, in the limit this expression simplifies tremendously. As signals are distributed uniformly on $[0, v]$, conditioned on $s_{i}=Y_{-i}^{n}\left(k_{n}\right)=s$ one can say with near certainty that $v=2 s$. Moreover, in the limit $G\left(Y_{-i}^{n}\left(k_{n}\right)=s \mid s_{i}=s\right) \rightarrow \frac{s}{1-s}$, while $g\left(Y_{-i}^{n}\left(k_{n}\right)=s \mid s_{i}=s\right) \rightarrow \frac{2}{1-s}$. Hence, (1) simplifies to

$$
b^{\prime}(s) \frac{s}{1-s}= \begin{cases}\frac{2}{1-s}(2 s-b(s)) & \text { if } s<.5 \\ 0 & \text { if } s_{i} \geq .5 .\end{cases}
$$

The fact that the derivative $b^{\prime}(s)=0$ for $s>0.5$ follows from the fact that in the limit the median is lower than 0.5 with certainty, and so raising one's bid only increases the payment. This is the key to our analysis, as bids flatten out in a discriminatory auction (but not in a uniform price auction), and are never above conditional expected value to begin with. Using the boundary condition of $b(0)=0$, we get the following characterization for the limiting bid function.

$$
b(s)= \begin{cases}\frac{4 s}{3} & \text { if } s<.5, \text { and } \\ \frac{2}{3} & \text { if } s_{i} \geq .5\end{cases}
$$

Consider two cases,

\footnotetext{
${ }^{4}$ This violates (A3) as $g_{S_{i} \mid V}$ is not continuous (at $s=v$ ), and also violates (A5) as it only satisfies a weak inequality and is discontinuous at $s=v$. However, the example is very easy to solve and thus makes the intuition transparent. The example if perturbed slightly to satisfy our assumptions. Finally, the example also illustrates that our conclusions extend beyond the specific assumptions here.
} 
- $v \leq 0.5$ - In this case winning bidders are uniform on $[v / 2, v]$ with the average signal is $\frac{3 v}{4}$. Since in this range the bidding strategy is linear, we conclude that the average price converges to $\frac{3}{4} * \frac{4}{3} v=v$. Hence, the average price converges to the asset's value; and

- $v>0.5$ - In this case winning bidders are uniform on $[v / 2, v]$ with the average signal is $\frac{3 v}{4}$. However, in this range the bidding strategy is concave rather than linear. Hence, it follows that the average price converges to a number strictly below $v$.

The fact that bidding strategy flattens out for higher signals is the key reason why average prices are biased downward in the discriminatory auction. ${ }^{5}$

In this example the average price for low values converges to be exactly the value. That is partly an artifact of the structure of this particular example, as we already discussed in the remarks following the theorem, as it is possible for the price to exceed value at low values.

\section{Appendix}

The following lemma is useful in the proof of Theorem 1.

Lemma 3 Under (A1) through (A6)

(i) $g_{Y_{n} \mid S_{i}}(s \mid s)$ is uniformly bounded above across $n$ and $s$, and

(ii) Given any $a>s^{*}(0), G_{Y_{n} \mid S_{i}}(s \mid s)$ is uniformly bounded below across $n$ and $s>a$.

\section{Proof of Lemma 3:}

(i) Note that $g_{Y_{n} \mid S_{i}}(s \mid s)=\int g_{Y_{n} \mid v}(s \mid v) g(v \mid s) d v$.

Let us argue that $g_{V \mid S_{i}}(v \mid s)$ is bounded above. $g(v \mid s)=\frac{g(s \mid v) f(v)}{\int g(s \mid v) f(v) d v}$. Given that $g(s \mid v)>0$ for some $v$ by (A4) and this is continuous by (A3), the denominator is positive for all $s$. Moreover, as it is continuous in $s$ by (A2) and (A3), and positive everywhere on a compact set of $s$, it is uniformly bounded below in $s$. Given the continuity of the numerator in $s$ and $v$, it is bounded above uniformly on a compact set. The claim that $g_{V \mid S_{i}}(v \mid s)$ is bounded above then follows.

\footnotetext{
${ }^{5} \mathrm{On}$ average the revenue per unit converges to .43 in the discriminatory auction, compared to 0.5 in the uniform auction.
} 
So, it is enough to show that we can find $M$ so that $\int g_{Y_{n} \mid v}(s \mid v) d v<M$. Note that $g_{Y_{k_{n}} \mid V}(s \mid v)=\frac{n !}{(k-1) !(n-k) !} G_{S_{i} \mid V}(s \mid v)^{k_{n}-1}\left(1-G_{S_{i} \mid V}(s \mid v)\right)^{n-k_{n}} g_{S_{i} \mid V}(s \mid v)$, since $g_{S_{i} \mid V}(s \mid v)$ is bounded from above it is enough to consider:

$$
\int \frac{n !}{(k-1) !(n-k) !} G_{S_{i} \mid V}(s \mid v)^{k_{n}-1}\left(1-G_{S_{i} \mid V}(s \mid v)\right)^{n-k_{n}} d v
$$

We change variables by letting $X(v)=G_{S_{i} \mid V}(s \mid v)$ and get

$$
\int \frac{n !}{(k-1) !(n-k) !} X^{k_{n}-1}(1-X)^{n-k_{n}} d X(v)
$$

Let us argue that $X^{\prime}(v)$ is bounded above and below. Note that under this change of variables, $\frac{\partial G_{S_{i} \mid V}(s \mid v)}{\partial v}$ is nonpositive under (A5), and so is bounded above by 0 . It is bounded below as follows.

$$
G(s \mid v)-G\left(s \mid v^{\prime}\right)=\int_{0}^{s}\left[g\left(s^{\prime} \mid v\right)-g\left(s^{\prime} \mid v^{\prime}\right)\right] d s^{\prime} .
$$

Given the continuity of $\frac{\partial g\left(s^{\prime} \mid v\right)}{\partial v}$ on a compact set, we can find $A$ such that

$$
g\left(s^{\prime} \mid v\right)-g\left(s^{\prime} \mid v^{\prime}\right)<A\left(v-v^{\prime}\right),
$$

and the result then follows.

So, it is enough to consider

$$
\int \frac{n !}{(k-1) !(n-k) !} X^{k_{n}-1}(1-X)^{n-k_{n}} d x
$$

Note that the integrand above is the density of the $k_{n}$ order statistic evaluated at $X$, where the sample is drawn from the uniform distribution. Hence the integral is one.

(ii) Fix $a>s^{*}(0)$. By the continuity of $G_{V \mid S_{i}}$ (from (A2) and (A3)), it follows from (A6) that there exists $\delta>0$ such that $G_{V \mid S_{i}}(v(s) \mid s)>\delta$ for any $s \geq a$. Since $g_{V \mid S_{i}}$ is uniformly bounded above (based on an argument similar to that behind (i)), we can find some $\delta_{1}>0$ so that $G_{V \mid S_{i}}\left(v(s)-\delta_{1} \mid s\right)>\delta_{1}$ for all $s \geq a$.

Next, let us argue that the strict MLRP condition (A5) implies that for any $v \leq$ $v(s)-\delta_{1}$ there exists $\delta_{2}>0$ so that $G(s \mid v)>1-\gamma+\delta_{2}$. Let $v^{\prime}=v(s)-\delta_{1}$. It is enough to bound $\frac{1-G\left(s \mid v^{\prime}\right)}{G\left(s \mid v^{\prime}\right)}$ away from $\frac{1-G(s \mid v(s))}{G(s \mid v(s)}=\frac{\gamma}{1-\gamma}$. Note that $\frac{1-G\left(s \mid v^{\prime}\right)}{G\left(s \mid v^{\prime}\right)}=\frac{\int_{s}^{1} g\left(t \mid v^{\prime}\right) d t}{\int_{0}^{s} g\left(t \mid v^{\prime}\right) d t}, \frac{1-G(s \mid v(s))}{G(s \mid v(s))}=$ $\frac{\int_{s}^{1} g(t \mid v(s)) d t}{\int_{0}^{s} g(t \mid v(s)) d t}$ Fix an $\varepsilon$ and note that A5 implies that $\frac{g\left(t_{1} \mid v^{\prime}\right)}{g\left(t_{2} \mid v^{\prime}\right)}>\frac{g\left(t_{1} \mid v((s))\right.}{g\left(t_{2} \mid v(s)\right)}(1+\tau)$ for some $\tau>0$ for any $t_{1}, t_{2}$ so that $t_{1} \geq t_{2}+\varepsilon$ (where we are using the continuity of the partial derivative on a compact set). This implies that

$$
\frac{\int_{s+\varepsilon}^{1} g\left(t \mid v^{\prime}\right) d t}{\int_{0}^{s} g\left(t \mid v^{\prime}\right) d t}>(1+\tau) \frac{\int_{s+\varepsilon}^{1} g(t \mid v(s)) d t}{\int_{0}^{s} g(t \mid v(s)) d t}
$$


The reason why the above is true is that if $\frac{A_{i}}{B_{j}}>\frac{a_{i}}{b_{j}}(1+\tau)$ for any $i, j$, then $\frac{A_{i}}{\sum_{j} B_{j}}>$ $(1+\tau) \frac{a_{i}}{\sum_{j} b_{j}}$, which implies that $\frac{\sum_{i} A_{i}}{\sum_{j} B_{j}}>(1+\tau) \frac{\sum_{i} a_{i}}{\sum_{j} b_{j}}$. Also note that (A5) implies

$$
\frac{\int_{s}^{s+\varepsilon} g\left(t \mid v^{\prime}\right) d t}{\int_{0}^{s} g\left(t \mid v^{\prime}\right) d t}>\frac{\int_{s}^{s+\varepsilon} g(t \mid v(s)) d t}{\int_{0}^{s} g(t \mid v(s)) d t} .
$$

The claim then follows, as we can pick $\varepsilon$ to be small, and this combined with $g(s \mid v)$ being uniformly bounded implies that we can make $\int_{s}^{s+\varepsilon} g(t \mid v(s)) d t$ small.

Using Chebyshev's bound we conclude that conditional on $v \leq v(s)-\delta_{1}$, the probability that $s \geq Y_{n}$ is bounded away from zero across $n$ and $s \geq a$. To see this, we argue that $\operatorname{Pr}\left(Y_{n} \leq s \mid V=v\right)$ is bounded away from zero across $s$ and $v$ such that $G(s \mid v)>1-\gamma+\delta_{2}$. Consider $n$ i.i.d Bernoulli random variables $\left\{X_{i}\right\}_{i=1}^{n}$ where $\operatorname{Pr}\left(X_{i}=1\right)=p \equiv 1-\gamma+\delta_{2}$. Let $X=\sum X_{i}$. Then

$$
\operatorname{Pr}\left(Y_{n} \leq s \mid V=v\right) \geq \operatorname{Pr}(X \geq n(1-\gamma))
$$

Note that $E X=\left(1-\gamma+\delta_{2}\right) n$ and $\operatorname{var}(X)=n p(1-p)$. We note also that $\operatorname{Pr}(X<(1-\gamma) n)=$ $\operatorname{Pr}\left(X<E X-\delta_{2} n\right)$. Chebyshev's bound then implies that

$$
\operatorname{Pr}\left(X<E X-\delta_{2} n\right) \leq \frac{\operatorname{var}(X)}{\left(\delta_{2} n\right)^{2}}=\frac{1}{n} \frac{\left(1-\gamma+\delta_{2}\right)\left(\gamma-\delta_{2}\right)}{\left(\delta_{2}\right)^{2}}
$$

which is bounded away from 1, which means that $\operatorname{Pr}(X \geq n(1-\gamma))$ is bounded away from 0. Thus, so is $\operatorname{Pr}\left(Y_{n} \leq s \mid V=v\right)$. Part (ii) now follows since $G_{V \mid S_{i}}\left(v(s)-\delta_{1} \mid s\right)>$ $\delta_{1}$.

Proof of Theorem 1: A direct extension of the results of Milgrom and Weber (1982) for first price auctions leads to the derivation of a symmetric equilibrium for the discriminatory auction as follows. If a type $s$ pretends to have a slightly higher signal, then the additional cost is $b_{n}^{\prime}(s)$ times the probability that $s$ was among the highest $k_{n}$ signals. The expected benefit from such a deviation is winning an object in the marginal event where $s$ was tied with the $k_{n}$-th signal. The probability of this is the density of the $k_{n}$-th highest signal at $s$ conditional on observing $s_{i}=s$. The benefit conditional on this event is the difference between value and bid. This leads to the following differential equation.

$$
b^{\prime}(s) G\left(Y_{-i}^{n}=s \mid S_{i}=s\right)=g\left(Y_{-i}^{n}=s \mid S_{i}=s\right)\left(v_{n}(s, s)-b(s)\right)
$$

where $v_{n}(t, s) \equiv E\left(V \mid Y_{-i}^{n}=t, S_{i}=s\right)$. The solution to the above differential equation combined with the boundary condition $b_{n}(0)=0$ yields a pure strategy, increasing, symmetric equilibrium.

We first prove the convergence claim for signals that in the limit expect to receive an object with probability that is strictly between zero and one. That is, we consider 
signals $s \in\left(s^{*}(0), s^{*}(1)\right)$ and argue that

$$
b_{n}(s) \rightarrow v(s)-\int_{s^{*}(0)}^{s} L(\alpha \mid x) d t(\alpha),
$$

where

$$
L(\alpha \mid x)=e^{-\int_{\alpha}^{x} g_{Y \mid S_{i}}(s \mid s) / G_{Y \mid S_{i}}(s \mid s) d s},
$$

$t(\alpha)=v(\alpha), v(s)=\lim _{n} v_{n}(s, s)=\left[s^{*}\right]^{-1}(s)$, and where we take $L$ to be 0 whenever the integral diverges. ${ }^{6}$

By the same reasoning as in Milgrom and Weber (1982), for a given $n$ we can express an equilibrium bidding function as:

$$
b_{n}(s)=b_{n}\left(s^{*}(0)\right)+v_{n}(s, s)-v_{n}\left(s^{*}(0), s^{*}(0)\right)+\int_{s^{*}(0)}^{s} L_{n}(\alpha \mid x) d t_{n}(\alpha),
$$

where $t_{n}(\alpha)=v_{n}(\alpha, \alpha)$ and

$$
L_{n}(\alpha \mid x)=e^{-\int_{\alpha}^{x} g_{Y_{n} \mid S_{i}}(s \mid s) / G_{Y_{n} \mid S_{i}}(s \mid s) d s} .
$$

Given the convergence of $v_{n}(s, s)$ to $v(s)$, we need only check that

$$
\int_{s^{*}(0)}^{s} L_{n}(\alpha \mid x) d t_{n}(\alpha) \rightarrow \int_{s^{*}(0)}^{s} L(\alpha \mid x) d t(\alpha)
$$

for any $s \in\left(s^{*}(0), s^{*}(1)\right)$.

Consider any $\alpha, x \in\left(s^{*}(0), s^{*}(1)\right)$. We know that $g_{Y_{n} \mid S_{i}}(s \mid s) \rightarrow g_{Y \mid S_{i}}(s \mid s)$ for any $s>s^{*}(0)$ (where $g_{Y \mid S_{i}}(s \mid s)=g_{V \mid S_{i}}(v(s) \mid s)$ ), $G_{Y_{n} \mid S_{i}}(s \mid s) \rightarrow G_{Y \mid S_{i}}(s \mid s)$ for any $s>s^{*}(0)$ (where $G_{Y \mid S_{i}}(s \mid s)=G_{V \mid S_{i}}(v(s) \mid s)$ ), and the $g_{Y_{n} \mid S_{i}}$ 's are bounded above uniformly and the $G_{Y_{n} \mid S_{i}}$ are bounded away from zero uniformly (across $s>\alpha$, noting $\alpha>s^{*}(0)$ ) by Lemma 3. Therefore, we can apply the Dominated Convergence Theorem to conclude that $L_{n}(\alpha \mid x) \rightarrow L(\alpha \mid x)$. Note that $L_{n}(\alpha \mid x)$ is nonnegative and bounded uniformly above $(\operatorname{across} n)$ by $e^{-0}=1$. We also know that that $t_{n}(\alpha)$ converges to $t(\alpha)$ and that these are bounded above uniformly. We can apply the Dominated Convergence Theorem again to conclude that

$$
\int_{s_{*}}^{s} L_{n}(\alpha \mid s) d t_{n}(a) \rightarrow \int_{s_{*}}^{s} L(\alpha \mid s) d t(a)
$$

for any $s \in\left(s^{*}(0), s^{*}(1)\right)$, as claimed. ${ }^{7}$

\footnotetext{
${ }^{6}$ The $Y$ is the limit of the $Y_{n}$ 's, which becomes a degenerate random variable as a function of $V$ in the limit.

${ }^{7}$ We need to be a bit careful as both the functions and the measures of integration are changing with $n$. However, both are bounded and converging and so a variation on the Dominated Convergence Theorem can be used. In particular, let $\left\{h_{n}\right\},\left\{j_{n}\right\}$ be two sequences of functions such that (i) $0 \leq h_{n} \leq j_{n}$, (ii) $h_{n} \rightarrow h, j_{n} \rightarrow j$ almost everywhere, and let $d \mu_{n}$ be a sequence of measures that converge set wise to a measure $d \mu$, then $\lim \int j_{n} d \mu=\int j d \mu$ implies $\lim \int h_{n} d \mu_{n}=\int h d \mu$. This follows from a generalized version of Fatou's Lemma that appears in Royden p. 269. This lemma implies that $\lim \inf \int h_{n} d \mu_{n} \geq$ $\int h d \mu$, hence it is enough to show that limsup $\int h_{n} d \mu_{n} \leq \int h d \mu$. Since $j_{n}-h_{n} \geq 0$ we can apply again the generalized version of Fatou's Lemma to conclude that $\lim \inf \int j_{n}-h_{n} d \mu_{n} \geq \int j-h d \mu$. However, $\liminf \int j_{n}-h_{n} d \mu_{n}=\int j d \mu-\limsup \int h d \mu_{n}$. hence, indeed $\lim \sup \int j_{n} d \mu \leq \int h d \mu$.
} 
By the definition of $h$ it follows that

$$
h(s)=\frac{\int_{y=0}^{s} v(y) g_{Y \mid s}(y \mid z) d z}{G_{Y \mid s}(s \mid s)} .
$$

Thus,

$$
h^{\prime}(s)=\frac{g_{Y \mid s}(s \mid s)}{G_{Y \mid s}(s \mid s)}(v(s)-h(s)) .
$$

Again (as in Theorem 14 in Milgrom and Weber (1982)) we can write

$$
h(s)=v(s)-\int L(\alpha \mid x) d t(\alpha) .
$$

This is the same as the expression for $\lim _{n} b_{n}(s)$, and thus establishes the claim.

Next, we consider $s>s^{*}(1)$, note that $s^{*}(1)<1$. Since the bidding function is increasing in $s_{i}$, it follows that $s^{*}(1)$ is the asymptotic cutoff conditional on $V=1$ for getting an object. The MLRP and the strong law of large numbers imply that in equilibrium agents are "guaranteed" (with a probability converging to 1 as $n$ grows) to win an object if their signal is above $s^{*}(1)$. This implies that in a discriminatory auction the $b_{n}\left(s_{i}\right)$ becomes flat on $\left[s^{*}(1), 1\right]$. That is, $b_{n}\left(s_{i}^{\prime}\right)-b_{n}\left(s_{i}\right) \rightarrow_{n} 0$ for any $s_{i}^{\prime}>s_{i}>s^{*}(1)$. This follows since for large enough $n$, an agent who has signal $s_{i}^{\prime}$ can bid as if she had signal $s_{i}$ with an arbitrarily small impact on the probability of getting an object, but a savings of the difference in bids in terms of price paid conditional on winning.

\section{References}

Bali, V. and M.O. Jackson (2002), "Asymptotic Revenue Equivalence," Journal of Economic Theory, Vol. 106, pp 161-176.

Jackson, M.O. and I. Kremer (2003a), "The Relevance of the Choice of an Auction Format in a Competitive Environment," mimeo: California Institute of Technology and Stanford University.

Jackson, M.O. and I. Kremer (2003b), "The Relationship between the Allocation of Goods and a Seller's Revenue," forthcoming: Journal of Mathematical Economics.

Milgrom, P. (1979), "A Convergence Theorem for Competitive Bidding with Differential Information," Econometrica, Vol. 47, pp. 670-688. 
Milgrom, P. (1981), "Rational Expectations, Information Acquisition, and Competitive Bidding," Econometrica, Vol. 49, pp. 921-944.

Milgrom, P. (1981a), "Good News and Bad News: Representation Theorems and Applications," The Bell Journal of Economics, Vol. 12, pp. 380-391.

Milgrom, P. and R. Weber (1982), "A Theory of Auctions and Competitive Bidding," Econometrica, Vol. 50, pp. 1089-1122.

Pesendorfer, W. and J. Swinkels (1997), "The Loser's Curse and Information Aggregation in Common Value Auctions," Econometrica, Vol. 65, pp. 12471282.

Wilson, R. (1977), "A Bidding Model of Perfect Competition," Review of Economic Studies, Vol. 4, pp. 511-518. 